\title{
On the Laws of Personal Information Protection in Network Environment
}

\author{
Xin-ya Chen ${ }^{\mathrm{a}}$ and Yue-tong Liu ${ }^{\mathrm{b} *}$
}

\author{
University of Electronic Science and Technology of China, School of Political Science and Public \\ Administration, Chengdu, Sichuan, P.R. China \\ aliuyuetong916@163.com, ${ }^{\text {b2 } 275262026 @ q q . c o m ~}$
}

Keywords: Network environment, Personal information rights, Protection, Measures

\begin{abstract}
With the increasing significance of the Internet in people's lives, the infringement of personal information in the network environment is aggravated. The infringement is mainly classified into the following four types: infringement of personal data and information, violation of personal online privacy, and disturbance of personal peace of life. Currently, the Civil Law of the People's Republic of China, the Cybersecurity Law of the People's Republic of China, the Criminal Law and other administrative regulations contain provisions in this regard. However, problems such as poor operability and fewer rights relief channels hinder the enforcement. A solution is that a Personal Information Protection Act should be introduced to make the existing laws comprehensive and systematic.
\end{abstract}

\section{Introduction Research Question and Overview}

On August 21, 2016, an 18-year-old girl Xu Yuyu, who had just finished her college entrance examination, found her college tuition RMB 9,900 swindled in a telephone scam and died after suffering a sudden cardiac arrest following invalid rescue in hospital. The telephone was made because her examination information was disclosed. Due to the sensational and malignant event, the cry for personal information protection reached the boiling point in society.

There is no clear definition of personal information in the legal profession, and there are three main views in the theoretical community. The first one is relevance-based, which suggests that people-related data, judgments and evaluations all belong to personal information; the second view is privacy-based, which suggests that personal sensitive information that individuals do not want to be known to those other than their relatives and friends is personal information; the third view is identification-based, which suggests that the name, age, ID number, home address, and other information of an individual that indicates his or her characteristics, individually or comparably to other information, are personal information [1]. The author agrees on the third view as it effectively avoids the generalization of the first definition that makes it hardly feasible while practically distinguishing personal information from privacy: identification is a necessary condition for personal information, while privacy is particularly concerned about the "hidden" nature of the information. Not to disclose personal life is an independent decision protected by law.

With the rapid development of network technology, there have been 688 million Chinese Internet users, and the Internet population rate has reached 50.3\%. People use the Internet to search, exchange and communicate information. As the social efficiency is greatly improved and lifestyles are changed, the barrier for personal information protection is gradually broken and personal information is easily made available through the Internet platform. The impact of the network environment on personal information protection is increasingly obvious and poses unprecedented challenge. In order to prevent further infringement of the personal information rights, resorting to legal force to a greater extent is the fundamental solution [2]. This paper is a beneficial attempt to further promote and improve the protection of personal information rights in the network environment.

\footnotetext{
* The two authors are equally contributed to this paper and both considered as first author. The names are sequenced alphabetically.
} 


\section{Infringement Behaviors of Personal Information Rights in the Network Environment}

The infringement of personal information rights in the network environment has various behavioral forms. The author classifies these behaviors into four categories based on the specific target.

\subsection{Infringement of personal data.}

In recent years, with the rise and development of the Internet of Things, personal data have been increasingly available on the Internet. Hackers also intend to get a share of this huge cake of benefits. Over time, their target has shifted from credit card accounts to personal data. Equifax, one of the largest credit agencies in the United States, collects information records of 160 million users and sells them to 50,000 businesses to make huge profits. Such stealing and trafficking of personal data makes the already fragile network-based personal data system more insecure and poses a huge risk to the protection of personal information.

\subsection{Invasion of personal privacy on the Internet.}

Although the use of database for analysis can improve the efficiency of selection and better meet the needs of users, during this process the users are not given the right to choose: whether to provide their data voluntarily; what to provide and what to not; what kinds of ad they would or would not accept. Deprived of the right to choose, the users do not realize how their information is collected by the sites or to what extent it is used. Their right to know the information is violated. There are some sites that use the bundled information and service model under which they refuse to provide services if users refuse to fill out the personal information. It seems that the decision is left for the users to made, yet they are actually forced to provide their detailed personal information, including name, height, marital status, family income, education background, health records and more.

\subsection{Disturbance of personal peace of life.}

It occurs frequently that: a flood of training agency ads pop up after we register for an examination online; we are overwhelmed by calls from decoration companies after buying a house, and they know clearly about our house type, location and area. As one of the most important contact means in the Internet era, E-mail should be one of the conveniences for individual life and work. However, the reality is that our mailboxes are often occupied by insurance ads and other commercials, and some virus e-mails are so indelible that they seriously waste the recipients' storage space and greatly undermine their peace of life and work efficiency.

\subsection{Others.}

In view of the various behaviors in infringing the personal information rights in the network environment, they are not enumerated here. Other acts that violate personal information rights in the network environment according to law are infringements.

\section{Current Legal Protection for Personal Information Rights on the Internet and Existing Problems}

\subsection{Legislation for personal information protection on the Internet in China}

\subsubsection{The basic civil law's protection for personal information on the Internet.}

The Civil Law of the People's Republic of China, as the opening chapter of the Chinese civil code, clarifies the ownership of personal information and emphasizes that information is personal property owned and decided by individuals who have the right to protect their own information. Article 111 in Chapter V Civil Rights stipulates that the personal information of natural persons is protected by law. Any organization or individual that needs personal information of others shall obtain and keep the information safe lawfully and shall not illegally collect, use, process or transmit 
such personal information of others or illegally trade, provide or disclose it. This is for the first time when personal information is written in law as a right to be protected.

\subsubsection{The protection for personal information on the Internet in single laws.}

The Cybersecurity Law of the People's Republic of China, as a basic law in the cyberspace field in China, explicitly stipulates to strengthen the protection of personal information in Chapter IV, "Information Security on the Internet". This law will further strengthen the protection for personal information, especially e-commerce data, in the network environment. In addition, Article 285 to Article 287 of the Criminal Law of the People's Republic of China set forth the criminal offenses of sabotaging computer information systems, dereliction of duty on the Internet, refusing to perform the obligations of network information security management, illegally using information on the Internet, and assisting in network information-related crimes, with a view to punish violations of the Internet-based personal information rights in serious circumstances.

\subsubsection{The protection for personal information on the Internet in departmental rules and local laws.}

For example, Article 9 and 10 of the Regulations on the Protection of Personal Information of Telecommunication and Internet Users promulgated by the Ministry of Industry and Information Technology of the People's Republic of China, Article 12 of the Regulations on the Management of Internet Electronic Bulletin Services issued by the Ministry of Industry and Information Technology. In addition, local governments in China have made efforts to protect personal information in the network environment in local laws and regulations, such as Article 38 of the Interim Measures for the Administration of Internet Transactions in Hangzhou and Article 11 of the Regulations on the Administration of Security of Computer Information Systems in Guangdong Province.

\subsection{Problems in the existing legislation about protection for personal information in the Internet in China}

\subsubsection{Laws with different levels of effectiveness are not systematic.}

As mentioned above, the author finds that, firstly, personal information protection is mentioned sporadically in the laws and regulations formulated by the National People's Congress, the NPC Standing Committee, the Ministry of Industry and Information Technology, and local governments, with the authorities ranging from the organ of supreme power to executive authorities with different levels of effectiveness. Secondly, the laws are made by different departments, making scattering or even partial repetition inevitable problems. There is a lack of coherence and unification between each other, which is not conducive to a reasonable allocation of responsibility system.

\subsubsection{The infringing party is not defined infallibly.}

Due to the nature and limited length of Civil Law of the People's Republic of China, this stipulation is inevitably inadequately instructive although it is forward-looking enough. In contrast, the Cybersecurity Law of the People's Republic of China has a separate chapter VI to make up for this deficiency. However, the author finds that almost all the responsible entities specified in this chapter are network operators, that is, network owners, managers and service providers. In fact, the infringing parties of personal information on the Internet are not limited to network operators, but include third-party offenders, or the so-called hackers, who infiltrate other people's network devices through network technologies and illegally obtain their personal information. This law makes no mention of the legal liability of such third-party offenders. The same legislative gap is also found in a large number of departmental rules and local regulations.

\subsubsection{The civil relief channels are not operable.}

The recognition of personal information rights in the Civil Law of the People's Republic of China provides the basis and possibility for citizens to seek relief when their rights are infringed [2]. Judging from the status quo of existing legislation in China, however, it is difficult for citizens to 
take legal weapons to protect their personal information rights, although the law does permit them to do so. In the Cybersecurity Law of the People's Republic of China, the use of personal information is regulated mainly by defining the rights and obligations of network operators and the state network information authority's regulatory power over network operators. The network information authority is the main body to hold accountable, and the main infringers are network operators. The enforcement relies on the state network information authority to exercise power and ignores third-party infringers. The same problems exist with similar departmental rules and administrative regulations. The Civil Law of the People's Republic of China, on the other hand, has generalized provisions that are not easily referred by the judge even if a case is filed.

\section{Measures for Improving Personal Information Protection in the Network Environment in China}

The personal information protection in the network environment requires a comprehensive and loop long-lasting mechanism that strengthens regulation from laws and regulations to administrative departments and from network operators to network information providers. The author tries to make some suggestions from the legal point of view below.

\subsection{Establish a comprehensive and systematic legal protection system}

Firstly, a separate Personal Information Protection Act should be established. At present, more than 50 countries and regions in the world have formulated laws and regulations related to the protection of personal information. These laws mostly govern the collection of individual information, the responsibilities and obligations of controllers, and the rights of individuals involved. Separate legislation for personal information protection is necessary for two reasons. For one thing, if the protection for personal information in the Civil Law of the People's Republic of China is the principle and the fundamental requirement, the enactment of Personal Information Protection Act is the basic foundation. With the protection of this law, the relief sought by the victims of personal information infringement is feasible [3]. On the other hand, now the main parties to hold accountable according to the existing laws are network operators and network service providers, while infringing third parties, namely "hackers", are left at large. The enactment of Personal Information Protection Act can make up for the gap in the existing legislation.

Secondly, special laws and regulations on the protection of personal information in the network environment should be formulated. In the field of personal information protection, the violation of personal information protection is particularly conspicuous in the network environment. On the basis of the Personal Information Protection Act, the establishment of special laws and regulations that specifically address the network environment can regulate from top to bottom the protection of personal information in the network environment systematically. Although a separate Personal Information Protection Act may make up for certain legislative gaps, it belongs to the civil law domain whose legal subjects must be civil subjects with equal status. In reality, however, the infringers of personal information rights on the Internet right are not necessarily civil subjects, but administrative subjects. In this case, the Personal Information Protection Act does not apply. China has the Cybersecurity Law of the People's Republic of China, however, they are not complete under the context of personal information protection on the Internet. Only by formulating special laws and regulations on personal information protection can we improve the system of civil rights in legislation so that citizens can use the law as a weapon to defend their personal rights in all aspects.

\subsection{Set up a regulatory authority to protect personal information on the Internet}

Having a regulatory authority to protect personal information on the Internet is a universal measure taken in many countries. The Federal Personal Data Protection Act of Germany sets up a Data Protection Committee to supervise the handling of personal data by public authorities and appoint supervisors to oversee the handling of personal data by non-official agencies. The amendment to the Privacy Act passed by the United States in 1988 stipulates that any federal 
government agency involved in the comparisons of personal information must set up a Data Protection Committee to monitor and protect the comparisons of personal information [4].

At present, China does not have a regulatory department that specializes in the management of personal information on the Internet. Under the Ministry of Industry and Information Technology are two departments performing the relevant functions. One is the Network Security Administration. The other department is the Information and Communication Administration. However, the functions of these two departments are overlapping and unclear. The protection of personal information on the Internet is an urgent and arduous task. Therefore, the author suggests setting up a special network personal information regulatory department.

The proposed regulatory department dedicated to network personal information security management performs two functions. On the one hand, a clear enforcement authority is assigned to implement the laws and regulations during the establishment and improvement of legal system for network-based personal information protection, and it will supervise and impose administrative punishment actively instead of accepting passively complaints or reports from victims. On the other hand, such authority can urge network information collectors to strengthen the security construction of their own websites and provide technical support when necessary. Under the supervision and support of government departments, this issue is bound to be alleviated.

\subsection{Promote self-examination and self-discipline of information collectors on the Internet}

Information collectors on the Internet often play the role of website operators and network service providers at the same time. In such circumstances, the collection of information is inevitable; however, there are several problems. China should introduce relevant policies or regulations to ensure that network information collectors meet the following four requirements. Firstly, they must comply with relevant laws and regulations, shall not disclose users' personal information, and shall protect their personal information. Secondly, the purpose and scope of users' information collected shall be clearly informed, and what measures will be taken by the operators to protect such personal information shall be well communicated. Thirdly, users shall be allowed to choose whether to provide their personal information or not rather than being forced. Fourthly, in the agreements signed with users, the operators shall remind them of the risks they may face and shall not incorporate too many disclaimers.

\subsection{Strengthen the self-protection awareness of information providers in the network}

As far as the author is concerned, the safety awareness of network information providers should be enhanced from the following aspects. First of all, network information providers should protect their personal information. They should confirm that a network operator has a security mechanism before providing the information requested. Secondly, encryption and privacy software should be used where appropriate. Network information providers should have the awareness of taking stronger safety measures when providing more important or more private personal information. Finally, network information providers shall be aware of the personal information protection right they own and shall be able to exercise this right to prevent their personal information from being disclosed.

\section{Conclusion}

In today's society, the Internet has become more of a necessity for life, and people are increasingly dependent on it, making it increasingly difficult to circumvent the risks it poses. The protection of personal information rights in the network environment is a problem that legislators can not ignore. On the basis of the recognized personal information rights in the Civil Law of the People's Republic of China, China may learn from the experience of comparative laws to develop a legal system of personal information protection with Chinese characteristics. With the promulgation of the Cybersecurity Law as well as the regulations of different departments and local laws, the author looks forward to the introduction of a distributed and advanced Personal Information 
Protection Act.

\section{References}

[1] Wang Li-ming. Legal Protection of Personal Information: Centered on the Line between Personal Information and Privacy. Modern Law Science, 1001-2397 (2013) 04-0062-11. (in Chinese)

[2] Shi Jia-you. Legislation on Personal Information Protection in the Network Environment. Journal of Suzhou University, 1001-4403 (2012) 06-0085-12. (in Chinese)

[3] Huang Lan. International Comparison and Inspiration of Personal Information Protection. Intelligence Science, 1007-7634 (2014) 01-143-07. (in Chinese)

[4] Qi Ai-min. Rescuing Personality in the Information Society: General Principles of Personal Information Protection Law. Peking University Press, 2009: 79. (in Chinese) 